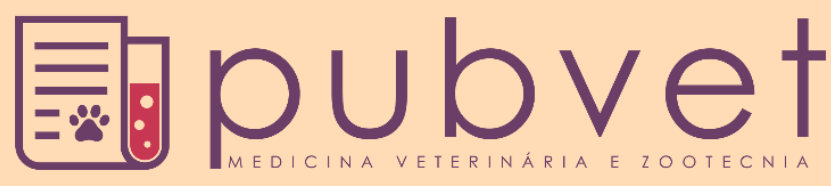

https://doi.org/10.31533/pubvet.v15n07a851.1-6

\title{
Análise do crescimento de Schizolobium parahyba var amazonicum (Huber Ex Ducke) Barneby sob diferentes proporções de pó-de-rocha
}

\author{
Hygor Gomes de Almeida Sousa ${ }^{1 *} \bullet$, Maristela Lima Figueiredo Guimarães Epifanio ${ }^{2} \bullet$ (D) , Igor \\ Viana Souza ${ }^{\circ}$, Bruno Aurélio Campos Aguiar $^{2}{ }^{\circ}$, Victor Braga Rodrigues Duarte ${ }^{10} \mathbb{D}$, \\ Gabriella Rayssa Antunes da Silva Oliveira ${ }^{3}{ }^{\circ}$ D, Marcos Vinicius de Melo Amorim ${ }^{3}{ }^{\circ}$, Camila Freire \\ Dias $^{4}{ }^{\bullet}$ (D) Flávia Bezerra de Souza ${ }^{1}$, Ana Clara Carneiro Fonseca ${ }^{1}{ }^{\circ}$, Priscila Bezerra de Souza ${ }^{5}$ \\ ${ }^{1}$ Discente do Programa de Pós-graduação em Ciências Florestais e Ambientais, da Universidade Federal do Tocantins, Campus de Gurupi-TO. Brasil \\ ${ }^{2}$ Mestre pelo Programa de Pós-graduação em Ciências Florestais e Ambientais, da Universidade Federal do Tocantins, Campus de Gurupi-TO. Brasil. \\ ${ }^{3}$ Discente do curso de Engenharia Florestal, da Universidade Federal do Tocantins, Campus de Gurupi - TO. Brasil. \\ ${ }^{4}$ Engenheira Florestal pela Universidade Federal do Tocantins, Campus de Gurupi-TO. Brasil. \\ ${ }^{5}$ Docente da Universidade Federal do Tocantins, Campus de Gurupi - TO. Brasil. \\ *Autor para correspondência, E-mail: hygoralmeida.floresta@outlook.com
}

Resumo. O Cerrado é um bioma do tipo biócoro, que ocupa cerca de $25 \%$ do território brasileiro, localizado principalmente na região central. O Schizolobium parahyba var. amazonicum (Huber ex Ducke) Barneby é uma espécie da família Fabaceae, de ocorrência em mata primária e secundária de terra firme da região amazônica. Esse trabalho tem como objetivo avaliar o desenvolvimento da espécie Schizolobium parahyba var. amazonicum diante de diferentes proporções de substrato alternativo. Foram utilizados 4 tratamentos, distribuídos da seguinte maneira, T1- Substrato Preparado (SP) 100\% (Terra preta, esterco bovino e palha de arroz), testemunha, T2- 80\% SP + 20\% Pó de Rocha (PR), T3- 65\% SP $+35 \%$ PR, T4-50\% SP + 50\% PR. O delineamento utilizado foi em blocos casualizados, com 5 repetições e os parâmetros utilizados foram: altura, diâmetro, teor de clorofila, massa seca da raiz (MSR), massa seca da parte aérea (MSPA), massa seca total (MST), razão massa seca da raiz e massa seca da parte aérea (MSR/MSPA), e índice de qualidade de Dickson. A análise de variância e as médias foram comparadas pelo teste de Tukey a 5\% de probabilidade através do programa Sisvar. Em todas as variáveis analisadas não houve diferença significativa para todos os tratamentos testados. O uso do pó de rocha como fertilizante não apresentou efeitos significativos no desenvolvimento da espécie estudada.

Palavras-chave: Cerrado, mudas, pó de rocha

\section{Growth analysis of schizolobium parahyba var amazonicum (huber ex ducke) barneby under different proportions of rock dustabstract.}

Abstract. The Cerrado is a biocore biome that occupies about $25 \%$ of the Brazilian
territory, located mainly in the central region. Schizolobium parahyba var. amazonicum
(Huber ex Ducke) Barneby is a species of the Fabaceae family, occurring in primary and
secondary terra firme forests of the Amazon region. This paper aims to evaluate the
development of the species Schizolobium parahyba var. amazonicum (Huber ex Ducke)
Barneby under different proportions of alternative substrate. Four treatments were used,
distributed as follows: T1- Prepared Substrate (SP) $100 \%$ (Black soil, cattle manure and
rice straw), control, T2- $80 \%$ SP $+20 \%$ Rock Powder (PR), T3- $65 \%$ SP $+35 \%$ PR, T4-
$50 \%$ SP + 50\% PR. The design used was randomized blocks with 5 replications and the
parameters used were: height, diameter, chlorophyll content, root dry mass (MSR), shoot
dry mass (MSPA), total dry mass (MST), ratio root dry mass and shoot dry mass (MSR / 
MSPA), and Dickson quality index. The analysis of variance and means were compared by Tukey test at 5\% probability using the Sisvar program. In all variables analyzed there was no significant difference for all treatments tested. The use of rock dust as fertilizer had no significant effects on the development of the studied species.

Keywords: Cerrado, seedlings, rock powder

\section{Introdução}

O Cerrado é um bioma do tipo biócoro, que ocupa cerca de $25 \%$ do território brasileiro, localizado principalmente na região central. O mesmo possui grande extensão territorial, sendo o segundo maior dentre os seis grandes biomas brasileiros, ficando atrás apenas da Floresta Amazônica, com a qual faz transição geográfica no estado do Tocantins compondo o domínio Extra Amazônico (Arruda et al., 2016). Este bioma tem fundamental importância ecológica para a região norte e suas províncias. Em toda a região norte há 156 Unidades Territoriais no nível de Unidades de Conservação Ambiental, entre Parques, Estações Ecológicas, Floretas Nacionais, Reservas Extrativistas ou Biológicas, Áreas de relevante Interesse Ecológico e Áreas de Proteção Ambiental (IBGE, 2019).

O estado do Tocantins guarda dois importantes ecótonos que constituem ricos laboratórios de estudos ambientais: o Ecótono-Cerrado Amazônia e o ecótono Cerrado-Caatinga. Estas áreas de transição alternam rica biodiversidade de estruturas vegetais, inseridas na região Hidrográfica Tocantins-Araguaia (Silva, 2007).

Contudo, a expansão da modernização das atividades agrícolas e da pecuária aumentam em até $80 \%$ os prejuízos causados às florestas. Estes dados são ainda intensificados pelo aumento da população. No estado do Tocantins há cerca de 1.550 .194 milhões de pessoas (IBGE, 2019) e a antropização contribui para a redução das Unidades de Conservação.

Com o intenso aumento da exploração desordenada do Cerrado para uso agropecuário e a diminuição dos recursos florestais, a produção de mudas de espécies florestais é uma alternativa para atender a demanda de produção para reflorestamento em áreas em processo de degradação (Lustosa Filho et al., 2015).

O Schizolobium parahyba var. amazonicum (Huber ex Ducke) Barneby é uma espécie da família Fabaceae, de ocorrência em Mata Primária e Secundária de Terra Firme da região amazônica, podendo ser utilizada como espécie para reflorestamento, áreas degradadas e sistemas agroflorestais que apresenta um bom valor comercial e se adapta facilmente a diferentes condições edafoclimáticas (Melo \& Menezzi, 2014). A espécie estudada possui rápido desenvolvimento, podendo chegar a $55 \mathrm{~cm} \mathrm{de}$ diâmetro à altura do peito (DAP), produzindo cerca de 150 a $340 \mathrm{~m}^{3} / \mathrm{ha}$ em apenas 15 anos. É, portanto, considerada uma espécie pioneira com ciclo de vida curto a médio (Ohashi et al., 2010). Tem como vantagens na sua produção o potencial de germinação e crescimento inicial rápido, com capacidade de fixação de nitrogênio no solo (Sarto et al., 2015), sendo apontada por Terezo et al. (2019) como uma escolha eficiente na composição de florestas recém plantadas para a região Norte e Centro-Oeste.

Devido ao baixo valor, os insumos não convencionais como pó de rochas Gnaisse, tem se tornado atraente por aprimorar as propriedades do solo e elevar a produção vegetal (Stappe \& Balloni, 1988). De acordo com Theodoro and Leonardos (2006), esse mineral é capaz de proporcionar quantidades de macronutrientes que podem ser agregados aos materiais orgânicos, tornando-se alternativa de insumo. $\mathrm{O}$ pó de rocha é um resíduo resultante das operações de mineração e apresenta grande potencial para locais com clima tropical, pois as taxas de dissolução desse mineral são favorecidas pela alta temperatura, além de reduzir custos relacionados a adubação (Wolschick et al., 2016).

Este trabalho tem como objetivo avaliar o desenvolvimento da espécie Schizolobium parahyba var. amazonicum em substrato alternativo composto por diferentes proporções de pó-de-rocha.

\section{Material e métodos}

O experimento foi realizado no Viveiro Florestal da Universidade Federal do Tocantins campus Universitário de Gurupi sob cobertura do tipo sombrite com $50 \%$ de retenção de luminosidade, no 
período de dois de maio de 2019 a dois de julho de 2019. A altitude da área experimental é de $287 \mathrm{~m}$, sob as coordenadas geográficas de latitude $11^{\circ} 43^{\prime} 45^{\prime \prime} \mathrm{S}$ e longitude $49^{\circ} 04^{\prime} 07^{\prime \prime} \mathrm{W}$ (Köppen \& Geiger, 1928). Segundo Köppen and Geiger (1928) o clima da região é do tipo AW, definido como tropical úmido com precipitação média anual entre $1.500 \mathrm{~mm}$ a $1.600 \mathrm{~mm}$ e temperatura média ao longo do ano entre 22 e $28^{\circ} \mathrm{C}$ (SEPLAN, 2012).

As mudas foram produzidas a partir de sementes adquiridas na empresa Arbocenter, com procedência de São Paulo. Para superação da dormência optou-se por escarificação mecânica. Efetuou-se a semeadura em tubetes de $290 \mathrm{~cm}^{3}$, sendo colocado duas sementes por tubetes. O delineamento utilizado foi em blocos casualizados, com cinco repetições, na qual a quantidade de repetições era a mesma quantidade de blocos e foram usadas três bandejas com 20 células ocupadas, totalizando 60 plantas. Foram utilizados quatro tratamentos, distribuídos da seguinte maneira, T1 - substrato preparado $100 \%$ (terra preta, esterco bovino e palha de arroz), testemunha, $\mathrm{T} 2-80 \% \mathrm{SP}+20 \%$ pó de rocha, $\mathrm{T} 3-65 \% \mathrm{SP}+35 \%$ pó de rocha, $\mathrm{T} 4-$ $50 \% \mathrm{SP}+50 \%$ pó de rocha. Após o plantio foi realizado duas irrigações diárias.

Trinta dias após a semeadura (DAS), foi realizado o desbaste, deixando apenas a plântula mais vigorosa e a que se encontrava na posição mais centralizada, a fim de favorecer o desenvolvimento da muda.

$\mathrm{O}$ experimento foi conduzido por 60 dias. Entretanto, mensalmente realizou-se medições de altura $(\mathrm{cm})$ utilizando régua graduada e diâmetro do colo $(\mathrm{mm})$ utilizando paquímetro digital, com o intuito de avaliar o desenvolvimento das mudas ao longo do experimento.

Ao final do experimento, coletou-se os dados de clorofila com o Clorofilog. Após, as mudas foram separadas em parte aérea e raiz e colocadas em sacos de papel kraft e alocados na estufa para secagem. As raízes foram lavadas para a retirada dos substratos. Todo o material foi seco em estufa com circulação de ar forçada a $60^{\circ} \mathrm{C}$ durante 72 horas. Posteriormente foi realizado a pesagem do material em balança analítica para obtenção da massa seca.

Os parâmetros utilizados foram: altura, diâmetro, teor de clorofila, massa seca da raiz (MSR), massa seca da parte aérea (MSPA), massa seca total (MST), razão massa seca da raiz e massa seca da parte aérea (MSR/MSPA), e partir desses parâmetros calculou-se índices de qualidade de mudas segundo a metodologia de Dickson et al. (1960).

Os dados obtidos foram submetidos a análise de variância e as médias comparadas pelo teste de Tukey a 5\% de probabilidade através do programa Sisvar (Ferreira, 2011).

\section{Resultados e discussão}

Os dados referentes a altura e diâmetro realizado aos 30 e 60 dias, em todos os tratamentos não apresentaram diferença significativa (Tabela 1). Resultados semelhantes foi encontrado por Suignard (2015), trabalhando com a mesma espécie Schizolobium parahyba var. amazonicum Barneby e os dados obtidos não diferiram estatisticamente entre si. De acordo com Gondin et al. (2015), substratos a base de subsolo, substrato orgânico comercial, palha de café e esterco de galinha agregado em concentrações específicas são capazes de serem promotores da redução de altura e diâmetro do caule das plantas com 30, 60 e 90 dias após a semeadura.

Tabela 1. Efeitos de diferentes composições de pó de rocha nos quatro tratamentos testados, dados de altura e diâmetro do coleto de mudas de Schizolobium parahyba var. amazonicum Barneby.

\begin{tabular}{lcccc}
\hline \multirow{2}{*}{ Tratamentos } & \multicolumn{3}{c}{30 dias } & \multicolumn{2}{c}{60 dias } \\
\cline { 2 - 5 } T1 & Altura, cm & Diâmetro, mm & Altura, cm & Diâmetro, mm \\
T2 & 29,64 & 3,52 & 31,34 & 4,01 \\
T1 & 27,70 & 3,48 & 30,73 & 3,92 \\
T4 & 28,52 & 3,54 & 30,46 & 4,12 \\
CV, \% & 29,64 & 3,64 & 29,17 & 4,06 \\
\hline
\end{tabular}

T1- Substrato Preparado 100\% (Terra preta, esterco bovino e palha de arroz), testemunha, T2 - 80\% SP + 20\% Pó de Rocha, T3 - 65\% SP + 35\% PR, T4 - 50\% SP + 50\% PR. Nível de 5\% de probabilidade pelo teste de Tukey $(\mathrm{P}<0,05)$.

Para as variáveis massa seca da raiz, massa seca da parte aérea, massa seca total e razão massa seca da raiz/massa seca da parte aérea não houveram diferenças $(\mathrm{P}>0,05)$ entre os tratamentos (Tabela 2$)$, 
corroborando com Araujo et al. (2014). Caron et al. (2010) ao trabalharem com Schizolobium parahyba var. parahyba, (Vell.) Blake utilizando o substrato comercial Tecnomax ${ }^{\circledR}$ não obtiveram diferença para as variáveis massa seca da raiz e parte aérea, dados estes que corroboram com o presente trabalho. Paiva et al. (2003), trabalhando com mudas de café, observaram que não houve efeito de biomassa no sistema radicial e parte aérea, conferindo esse acontecimento a capacidade da espécie ser estável na alocação energética durante seu desenvolvimento. Para a massa seca total encontrou-se resultados semelhantes aos apresentados por Araujo et al. (2014), ao testarem solo de mangue, areia e latossolo com a espécie Avicennia germinans var. guayaquilensis (Humb., Bonpl. \& Kunth) Moldenke.

A razão da massa seca da parte aérea e da massa seca da raiz não diferenciaram $(\mathrm{P}>0,05)$. Estes que corroboram com Siqueira et al. (2019), que ao estudarem a espécie Lafoensia glyptocarpa (Koehne, B.A.E) juntamente com o substrato comercial Basaplant Florestal e lodo de esgoto tratado distribuído em proporções, também não encontraram diferença. Cabe ressaltar que os tratamentos 1 e 2 apresentaram resultados próximos a 0,5 proposto como ideal por Caldeira et al. (2013), na qual aumentaria as chances de sobrevivência das mudas em um pós plantio. Gomes \& Paiva (2006) afirmaram que a massa seca da raiz pode ser avaliada como uma das melhores e mais importantes variáveis na estimativa da sobrevivência e crescimento inicial de mudas em campo.

Tabela 2. Efeitos das diferentes composições de pó de rocha nos quatro tratamentos testados, dados de massa seca de raiz (MSR), parte aérea (MSPA), massa seca total (MST) e razão raiz/parte aérea de mudas de Schizolobium parahyba var. amazonicum.

\begin{tabular}{lcccc}
\hline Tratamentos & MSR, $g$ & MSPA, $g$ & MST, $g$ & MSR/MSPA, g \\
\hline T1 & 0,453 & 0,781 & 1,234 & 0,601 \\
T2 & 0,473 & 0,737 & 1,210 & 0,667 \\
T3 & 0,562 & 0,787 & 1,349 & 0,777 \\
T4 & 0,536 & 0,730 & 1,266 & 0,730 \\
\hline CV, \% & 28,68 & 29,48 & 25,68 & 30,42 \\
\hline
\end{tabular}

Nível de $5 \%$ de probabilidade pelo teste de Tukey.

Segundo os resultados encontrados neste trabalho, percebe-se que não houve diferença estatística entre os tratamentos (Tabela 3). Resultados semelhantes também foram apresentados por Siqueira et al. (2019) ao avaliarem a espécie Lafoensia glyptocarpa (Koehne, B.A.E). O índice de qualidade de Dickson (IQD) é considerado um indicador capaz de expressar a qualidade das mudas por levar em conta diversos parâmetros simultaneamente, sendo que quanto maior o seu valor, maior é a qualidade das mudas (Siqueira et al., 2019).

Os valores de clorofila A e B, entre os tratamentos testados não houve diferença estatística (Tabela 3). A clorofila, além dos pigmentos acessórios, é fundamental à planta na captação de energia solar, eficiência, crescimento e adaptabilidade das plantas (Afonso et al., 2017). Corroboram com estes resultados o estudo de Cruz (2018), que ao trabalhar com as espécies Schizolobium parahyba var. parahyba e Schizolobium parahyba var. amazonicum e utilizar como substrato uma mistura de solo e adubo (esterco bovino) também não obteve diferenças estatísticas.

Tabela 3. Efeitos de diferentes composições de pó de rocha nos quatro tratamentos testados, dados de Índice de Qualidade de Dickison (IQD), clorofila A e B das mudas de Schizolobium parahyba var. amazonicum (Huber ex Ducke) Barneby.

\begin{tabular}{lccc}
\hline Tratamentos & IQD & Clorofila A & Clorofila B \\
\hline T1 & 0,122 & 23,523 & 4,644 \\
T2 & 0,129 & 22,251 & 4,704 \\
T3 & 0,142 & 22,787 & 4,989 \\
T4 & 0,142 & 23,523 & 4,662 \\
\hline CV $\%$ & 29,18 & 24,24 & 31,97 \\
\hline
\end{tabular}

Nível de 5\% de probabilidade pelo teste de Tukey $(\mathrm{P}<0,05)$.

Contudo, a influência do substrato em diferentes proporções pode alterar quantitativamente o teor de pigmentos fotossintéticos (Afonso et al., 2017), visto que o teor de clorofila nas folhas é de suma importância para aferir o potencial fotossintético das plantas, pelo seu vínculo direto com a absorção e transferência de energia luminosa que interferem no crescimento (Rego \& Possamai, 2006). 


\section{Conclusão}

Com base nos parâmetros analisados, a espécie Schizolobium parahyba var. amazonicum, não apresentou efeitos significativos diante das diferentes proporções de substrato utilizadas no desenvolvimento de mudas da espécie estudada em casa de vegetação.

As condições de desenvolvimento em casa de vegetação requerem que o substrato contenha proporções adequadas de materiais que sejam capazes de melhorar as características físicas e químicas do substrato. É possível sugerir, contudo, diante dos resultados em campo, na qual demonstram que o substrato utilizado já continha características físicas e químicas para o bom desenvolvimento da muda, justificando o fato de não se ter encontrado diferenças significativas.

\section{Referências}

Afonso, M. V., Martinazzo, E. G., Aumonde, T. Z., \& Villela, F. A. (2017). Parâmetros fisiológicos de mudas de Albizia niopoides produzidas em diferentes composições de substrato. Ciência Florestal, 27(4), 1395-1402. https://doi.org/10.5902/1980509830221.

Araujo, E. C., Costa, R. S., Lopes, E. C., Daher, R. F., \& Fernandes, M. E. B. (2014). Qualidade das mudas de espécies arbóreas de mangue cultivadas em viveiro e diferentes substratos. Revista Acta Ambiental Catarinense, 11(1/2), 21-32.

Arruda, H. S., Botrel, D. A., Fernandes, R. V. B., \& Almeida, M. E. F. (2016). Development and sensory evaluation of products containing the Brazilian Savannah fruits araticum (Annona crassiflora Mart.) and cagaita (Eugenia dysenterica Mart.). Brazilian Journal of Food Technology, 19, 1-7. https://doi.org/10.1590/1981-6723.10515.

Caldeira, M. V. W., Delarmelina, W. M., Faria, J. C. T., \& Juvanhol, R. S. (2013). Substratos alternativos na produção de mudas de Chamaecrista desvauxii. Revista Árvore, 37(1), 31-39. https://doi.org/10.1590/S0100-67622013000100004.

Caron, B. O., Souza, V. Q. de, Cantarelli, E. B., Manfron, P. A., Behling, A., \& Eloy, E. (2010). Crescimento em viveiro de mudas de Schizolobium parahyba (Vell.) SF Blake. submetidas a níveis de sombreamento. Ciência Florestal, 20(4), 683-689. https://doi.org/10.5902/198050982427.

Cruz, T. S. (2018). Divergência fenotípica em variedades do gênero Schizolobium por caracteres biométricos e fisiológicos (pp. 1-8). Universidade Federal do Recôncavo da Bahia.

Dickson, A., Leaf, A. L., \& Hosner, J. F. (1960). Quality appraisal of white spruce and white pine seedling stock in nurseries. The Forestry Chronicle, 36(1), 10-13. https://doi.org/10.5558/tfc362373.

Ferreira, D. F. (2011). SISVAR: A Computer Statistical Analysis System. Ciência e Agrotecnologia, 35(6), 1039-1042. https://doi.org/http://dx.doi.org/10.1590/S1413-70542011000600001.

Gomes, J. M., \& Paiva, H. N. (2006). Viveiros florestais: propagação sexuada. Universidade Federal de Viçosa.

Gondin, J. C., Silva, J. B., Alves, C. Z., Dutra, A. S., \& Elias Junior, L. (2015). Emergência de plântulas de Schizolobium amazonicum Huber ex Ducke (CAESALPINACEAE) em diferentes substratos e sombreamento. Revista Ciência Agronômica, 46(2), 329-338. https://doi.org/10.5935/18066690.20150012 .

IBGE. Indicadores de Desenvolvimento Sustentável. Disponível em: <https://sidra.ibge.gov.br/tabela/5629>. Acesso em: 02 out. 2019.

Köppen, W., \& Geiger, R. (1928). Klimate der Erde. Gotha: Verlag Justus Perthes. Wall-Map $150 \mathrm{~cm} \times 200 \mathrm{~cm}$.

Lustosa Filho, J. F., Nóbrega, J. C. A., Nobrega, R. S. A., Dias, B. O., Amaral, F. H. C., \& Amorim, S. P. N. (2015). Influence of organic substrates on growth and nutrient contents of jatob (Hymenaea stigonocarpa). African Journal of Agricultural Research, 10(26), 2544-2552. https://doi.org/10.5897/ajar2015.9781.

Melo, R. R., \& Menezzi, C. H. S. (2014). Influence of veneer thickness on the properties of LVL from Paricá (Schizolobium amazonicum) plantation trees. European Journal of Wood and Wood Products, 
72(2), 191-198. https://doi.org/10.1007/s00107-013-0770-8.

Ohashi, S. T., Yared, J. A. G., \& Farias Neto, J. T. (2010). Variabilidade entre procedências de paricá Schizolobium parahyba var amazonicum (Huber ex Ducke) Barneby plantadas no município de Colares-Pará. Acta Amazônica, 40(1), 81-88. https://doi.org/10.1590/s0044-59672010000100011.

Paiva, L. C., Guimarães, R. J., \& Souza, C. A. S. (2003). Influência de diferentes níveis de sombreamento sobre o crescimento de mudas de cafeeiro (Coffea arabica L.). Ciência $e$ Agrotecnologia, 27(1), 134-140. https://doi.org/10.1590/s1413-70542003000100016.

Rego, G. M., \& Possamai, E. (2006). Efeito do sombreamento sobre o teor de clorofila e crescimento inicial do Jequitibá-rosa. Pesquisa Florestal Brasileira, 53, 1-16.

Sarto, C., Segura, T. E. S., \& Silva Júnior, F. G. (2015). Performance of Schizolobium amazonicum wood in bleached kraft pulp production. BioResources, 10(3), 4026-4037. https://doi.org/10.15376/biores.10.3.4026-4037.

SEPLAN. Atlas do Tocantins: subsídios ao planejamento da gestão territorial, Palmas - Atual, v.6, 2012.

Silva, L. (2007). Biomas presentes no estado do Tocantins. In Consultoria Legilativa Nota Técnica Câmara dos Deputados, Brasilia, DF, Brasil (pp. 2-9).

Siqueira, D. P., Barroso, D. G., Carvalho, G. C. M. W., Erthal, R. M., Rodrigues, M. C. C., \& Marciano, C. R. (2019). Lodo de esgoto tratado na composição de substrato para produção de mudas de Plathymenia reticulata Benth. Ciência Florestal, 29(2), 728-739. https://doi.org/10.5902/1980509827297.

Stappe, J. L., \& Balloni, E. A. (1988). O uso de resíduos da indústria de celulose como insumos na produção florestal. Revista IPEF, 40, 33-37.

Suignard, Y. S. (2015). Emergência e crescimento de mudas de schizolobium parayba var. amazonicum (huber $x$ ducke) barneby em tubetes sob diferentes substratos. Universidade Federal do Recôncavo da Bahia.

Terezo, R. F., Córdova, F. O., \& Sampaio, C. A. P. (2019). Chemically treated glued laminated paricá timber (Schizolobium parahyba var. amazonicum). Engenharia Agrícola, 39(2), 158-165. https://doi.org/10.1590/1809-4430-Eng.Agric.v39n2p158-165/2019.

Theodoro, S. H., \& Leonardos, O. H. (2006). The use of rocks to improve family agriculture in Brazil. Anais Da Academia Brasileira de Ciências, 78(4), 721-730. https://doi.org/10.1590/s000137652006000400008.

Wolschick, P. R. D., Schuch, F. S., Gerber, T., \& Sartoretto, L. M. (2016). Efeito do pó de rocha basáltica sobre a germinação de Cedrela fissilis. Agropecuária Catarinense, 29(3), 76-80.

Histórico do artigo:

Recebido: 4 de dezembro de 2020

Aprovado: 15 de janeiro de 2021.
Licenciamento: Este artigoé publicado na modalidade Acesso Aberto sob a licença Creative Commons Atribuição 4.0 (CC-BY 4.0), a qual permite uso irrestrito, distribuição, reprodução em qualquer meio, desde que $o$ autor e a fonte sejam devidamente creditados. 